\title{
ON LIPSCHITZ AND D.C. SURFACES OF FINITE CODIMENSION IN A BANACH SPACE
}

\author{
LUDĚK ZAJÍČEK, Praha
}

\begin{abstract}
Properties of Lipschitz and d.c. surfaces of finite codimension in a Banach space, and properties of generated $\sigma$-ideals are studied. These $\sigma$-ideals naturally appear in the differentiation theory and in the abstract approximation theory. Using these properties, we improve an unpublished result of M. Heisler which gives an alternative proof of a result of D. Preiss on singular points of convex functions.
\end{abstract}

Keywords: Banach space, Lipschitz surface, d.c. surface, multiplicity points of monotone operators, singular points of convex functions, Aronszajn null sets.

MSC 2000: 46T05, 58C20, 47H05

\section{INTRODUCTION}

Let $X$ be a real separable Banach space. A number of $\sigma$-ideals of subsets of $X$ are considered in the literature. Besides the most classical system of first category sets mention the $\sigma$-ideals of Haar null sets, Aronszajn (equivalently Gaussian) null sets (see [2]), $\Gamma$-null sets (see [12, 11]) and $\sigma$-(lower or upper) porous sets (see e.g., 21]). In some questions of the differentiability theory and of the abstract approximation theory, the $\sigma$-ideals $\mathcal{L}^{1}(X)$ and $\mathcal{D} \mathcal{C}^{1}(X)$ generated by Lipschitz and d.c. Lipschitz hypersurfaces (i.e., "graphs" of Lipschitz and of d.c. Lipschitz functions), respectively, naturally appear. These $\sigma$-ideals are proper subsystems of all $\sigma$-ideals mentioned above. The sets from $\mathcal{L}^{1}(X)$ were used in $\mathbf{R}^{2}$ (under a different but equivalent definition) by W.H. Young (under the name "ensemble ridée") and by H. Blumberg (under the name "sparse set"); cf. [20, p. 294]. These sets were used in $\mathbf{R}^{n}$ e.g., (implicitly) by P. Erdös [4, and in infinite-dimensional spaces (possibly for the first time) in [18 and 17]. The sets from $\mathcal{D C}^{1}(X)$ were probably first applied in [19] (cf. 22, p. 93]). In some articles (e.g., 18, [19, 20, 15]) also sets from smaller $\sigma$-ideals $\mathcal{L}^{n}(X)$ and $\mathcal{D C}^{n}(X)$ generated by Lipschitz and d.c. Lipschitz surfaces of codimension $n>1$ were used.

In the present article we prove some properties of Lipschitz and Lipschitz locally d.c. surfaces of finite codimension (Section 3; Proposition 3.6 and Proposition 3.7).

Using these properties, we study in Section 4 sets which are projections of sets from $\mathcal{L}^{n}(X)$ on a closed space $Y \subset X$ of codimension $d<n$. The study of such projections was suggested by D. Preiss in connection with a result of [13] (see Remark 4.7(i)). M. Heisler [7] proved that any such projection is a first category set in $Y$, which provides (together with a result of [19]) an alternative proof of a result of $[13$. We prove that each such projection is also a subset of an Aronszajn null set in $Y$ (and even a subset of a set from a smaller class $\mathcal{C}_{n}^{*}$ ). As a consequence, we obtain a result on projections of sets of multiplicity of monotone operators (Theorem 4.9) which improves both [13, Theorem 1.3.] and the corresponding result of $[7$. 
Our proof is more transparent than that of 7 and gives stronger results, since it uses "perturbation" Proposition 3.7. To prove (and apply) it, we need some results on perturbations of finite-dimensional subspaces. These results are collected in Preliminaries, where also needful results on d.c. mappings are recalled.

\section{Preliminaries}

We consider only real Banach spaces. By $\operatorname{sp}\{M\}$ we denote the linear span of the set $M$. A mapping is called $K$-Lipschitz if it is Lipschitz with a (not necessarily minimal) constant $K$. A bijection $f$ is called bilipschitz ( $K$-bilipschitz) if both $f$ and $f^{-1}$ are Lipschitz ( $K$-Lipschitz).

A real function on an open convex subset of a Banach space is called d.c. (deltaconvex) if it is a difference of two continuous convex functions. Hartman's notion of d.c. mappings between Euclidean spaces [6] was generalized and studied in [16].

Definition 2.1. Let $X, Y$ be Banach spaces, $C \subset X$ an open convex set, and let $F: C \rightarrow Y$ be a continuous mapping. We say that $F$ is d.c. if there exists a continuous convex function $f: C \rightarrow \mathbf{R}$ such that $y^{*} \circ F+f$ is convex whenever $y^{*} \in Y^{*},\left\|y^{*}\right\| \leq 1$.

It is easy to see (cf. [16, Corollary 1.8.]) that, if $Y$ is finite dimensional, then $F$ is d.c. if and only if $y^{*} \circ F$ is d.c. for each $y^{*} \in Y^{*}$ (or for each $y^{*}$ from a fixed basis of $Y^{*}$ ). Note also that each d.c. mapping is locally Lipschitz (16, p. 10]). If $X$ is finite-dimensional, then each locally d.c. mapping is d.c. (see. [16, p. 14]) but it is not true (see [9]) if $X$ is infinite dimensional. We will need also the following well-known facts on d.c. mappings.

Lemma 2.2. Let $X, X_{1}, Y, Y_{1}, Y_{2}, Z$ be Banach spaces.

(i) Let $f: X \rightarrow Y$ be d.c. and let $g: X_{1} \rightarrow X, h: Y \rightarrow Y_{1}$ be linear and continuous. Then both $f \circ g$ and $h \circ f$ are d.c.

(ii) A mapping $f=\left(f_{1}, f_{2}\right): X \rightarrow Y_{1} \times Y_{2}$ is d.c. if and only if both $f_{1}$ and $f_{2}$ are d.c.

(iii) If $g: X \rightarrow Y, h: X \rightarrow Y$ are d.c. and $a, b \in \mathbf{R}$, then $a g+b h$ is d.c.

(iv) If $f: X \rightarrow Y$ is locally d.c. and $g: Y \rightarrow Z$ is locally d.c., then $g \circ f$ is locally d.c.

(v) Suppose that $G: X \rightarrow Y$ is a linear isomorphism, $g: X \rightarrow Y$ is a locally d.c. bilipschitz bijection, and the range of $g-G$ is contained in a finite dimensional space. Then $g^{-1}$ is locally d.c.

Proof. The statements (i) and (ii) are very easy (cf. [16, Lemma 1.5. and Lemma 1.7.]) and (iii) follows from (i) and (ii). The statement (iv) is a special case of [16, Theorem 4.2.] and (v) is a special case of [3, Theorem 2.1.].

We will need some notions and results concerning distances of two subspaces of a Banach space, which are well-known from the perturbation theory of linear operators $([5,[8,[1])$. Let $X$ be a Banach space and $S(X)$ be the unit sphere of $X$. Let $Y$ and $Z$ be closed non-trivial subspaces of $X$. Then the gap between $Y$ and $Z$ (called also the opening or the deviation of $Y$ and $Z$ ) is defined by

$$
\gamma(Y, Z)=\max \left\{\sup _{y \in Y \cap S(X)} \operatorname{dist}(y, Z), \sup _{z \in Z \cap S(X)} \operatorname{dist}(z, Y)\right\} .
$$

We set $\gamma(\{0\},\{0\}):=0$ and $\gamma(Y, Z)=1$ if one and only one of $Y, Z$ is $\{0\}$. The gap need not be a metric on the set of all non-trivial subspaces of $X$; this property has 
the distance $\rho(Y, Z)$ between $Y$ and $Z$ defined as the Hausdorff distance between $Y \cap S(X)$ and $Z \cap S(X)$.

We will work with the gap $\gamma(Y, Z)$. However, since it is easy to prove (see e.g., [8]) that (for nontrivial $Y, Z$ ) always

$$
\rho(Y, Z) / 2 \leq \gamma(Y, Z) \leq \rho(Y, Z),
$$

we could work also with $\rho(Y, Z)$. We will need the following well-known facts.

Lemma 2.3. Let $X$ be a Banach space and $F, \widetilde{F}, K$ be finite dimensional subspaces of $X$. Then:

(i) If $\gamma(F, \widetilde{F})<1$, then $\operatorname{dim} F=\operatorname{dim} \widetilde{F}$.

(ii) If $F \cap K=\{0\}$, then there exists $\omega>0$ such that $\gamma(F, \widetilde{F})<\omega$ implies $\widetilde{F} \cap K=\{0\}$.

(iii) If $E \oplus F=X$, then there exists $\omega>0$ such that $\gamma(F, \widetilde{F})<\omega$ implies $E \oplus \widetilde{F}=X$

Proof. Statement (i) is proved in [5] (see [1, Theorem 2.1]) and (ii) is an easy consequence of (2.1). (We can also apply [1, Theorem 5.2] with $Y:=F, Z:=K$ and $X:=F \oplus K$.) The statement (iii) immediately follows from [1, Theorem $5.2]$.

The following simple lemma is also essentially well-known. Although it is not stated explicitly in [10], it follows from [10, Theorem 2.2.] which works with complex Banach spaces. Since the formulation of [10, Theorem 2.2.] is rather complicated and we work with real spaces, for the sake of completness we give a proof.

Lemma 2.4. Let $X$ be a Banach space and $\left(v_{1}, \ldots, v_{n}\right)$ be a basis of a space $V \subset X$ and $\varepsilon>0$. Then there exists $\delta>0$ such that the inequalities $\| w_{1}-$ $v_{1}\|<\delta, \ldots,\| w_{n}-v_{n} \|<\delta$ imply that $W:=\operatorname{sp}\left\{w_{1}, \ldots, w_{n}\right\}$ is $n$-dimensional and $\gamma(V, W)<\varepsilon$.

Proof. First we will show that there exists $\eta>0$ and $\delta^{*}>0$ such that the inequality

$$
\left\|\sum_{i=1}^{n} c_{i} w_{i}\right\| \geq \eta\|c\|_{\infty}
$$

holds whenever $\left\|w_{1}-v_{1}\right\|<\delta^{*}, \ldots,\left\|w_{n}-v_{n}\right\|<\delta^{*}$ and $c=\left(c_{1}, \ldots, c_{n}\right) \in \mathbf{R}^{n}$ is arbitrary. To this end observe that there exists $\eta^{*}>0$ such that (2.2) holds for $\eta=\eta^{*}, w_{i}=v_{i}$, and arbitrary $c$. Put $\eta:=\eta^{*} / 2$ and $\delta^{*}:=\eta^{*} / 2 n$. Then the inequalities $\left\|w_{1}-v_{1}\right\|<\delta^{*}, \ldots,\left\|w_{n}-v_{n}\right\|<\delta^{*}$ imply that, for each $0 \neq c \in \mathbf{R}^{n}$,

$$
\left\|\sum_{i=1}^{n} \frac{c_{i}}{\|c\|_{\infty}} v_{i}\right\|-\left\|\sum_{i=1}^{n} \frac{c_{i}}{\|c\|_{\infty}} w_{i}\right\| \leq\left\|\sum_{i=1}^{n} \frac{c_{i}}{\|c\|_{\infty}}\left(v_{i}-w_{i}\right)\right\|<n \delta^{*}=\eta^{*} / 2 .
$$

Consequently, using the definition of $\eta^{*}$, we obtain

$$
\left\|\sum_{i=1}^{n} \frac{c_{i}}{\|c\|_{\infty}} w_{i}\right\| \geq\left\|\sum_{i=1}^{n} \frac{c_{i}}{\|c\|_{\infty}} v_{i}\right\|-\eta^{*} / 2 \geq \eta^{*}-\eta^{*} / 2=\eta,
$$

which implies (2.2). 
Now set $\delta:=\min \left\{\delta^{*}, \varepsilon \eta / 2 n\right\}$ and suppose that the inequalities $\left\|w_{1}-v_{1}\right\|<$ $\delta, \ldots,\left\|w_{n}-v_{n}\right\|<\delta$ hold. Let $w=\sum_{i=1}^{n} c_{i} w_{i}$ with $\|w\|=1$ be given. Set $v=\sum_{i=1}^{n} c_{i} v_{i}$. Since $\|c\|_{\infty} \leq 1 / \eta$ by (2.2), we obtain

$$
\|v-w\| \leq \sum_{i=1}^{n}\left|c_{i}\right| \delta \leq n(1 / \eta) \delta \leq \varepsilon / 2 .
$$

Consequently, $\sup _{w \in W \cap S(X)} \operatorname{dist}(w, V)<\varepsilon$. By a quite symmetrical way we obtain $\sup _{v \in V \cap S(X)} \operatorname{dist}(v, W)<\varepsilon$, so $\gamma(V, W)<\varepsilon$. Since we can suppose $\varepsilon<1$, we know that $W$ is $n$-dimensional by Lemma 2.3(i).

Lemma 2.5. Let $X, Y$ be Banach spaces and $F: X \rightarrow Y$ be a linear isomorphism. Then there exists $C>0$ such that

$$
C^{-1} \gamma(F(V), F(W)) \leq \gamma(V, W) \leq C \gamma(F(V), F(W)),
$$

whenever $V$ and $W$ are subspaces of $X$.

Proof. We can clearly suppose that $V$ and $W$ are non-trivial. Since $F^{-1}$ is also a linear isomorphism, it is clearly sufficient to find $D>0$ such that $\gamma(V, W) \leq$ $D \gamma(F(V), F(W))$ always holds. Choose $K>0$ such that $F$ is $K$-bilipschitz and consider $v \in V$ with $\|v\|=1$. We can clearly find $\widetilde{w} \in F(W)$ for which $\|\widetilde{w}-\| F(v) \|^{-1}$. $F(v) \| \leq 2 \gamma(F(V), F(W))$. Since $\|F(v)\| \leq K$, we have $\|F(v)-\| F(v)\|\cdot \widetilde{w}\| \leq$ $2 K \gamma(F(V), F(W))$, and therefore $\|v-\| F(v)\left\|\cdot F^{-1}(\widetilde{w})\right\| \leq 2 K^{2} \gamma(F(V), F(W))$. Since the roles of $V$ and $W$ are symmetric, we can clearly set $D:=2 K^{2}$.

Lemma 2.6. Let $X$ be an infinite dimensional Banach space, $V, W \subset X$ nontrivial finite dimensional spaces, and $\delta>0$. Then there exists a space $\widetilde{V} \subset X$ with $\gamma(V, \widetilde{V})<\delta$ and $\widetilde{V} \cap W=\{0\}$.

Proof. Denote $n:=\operatorname{dim} V$, choose an $n$-dimensional space $Y \subset X$ with $Y \cap(V+$ $W)=\{0\}$ and a linear bijection $L: V \rightarrow Y$. For $t>0$, set $\widetilde{V}_{t}:=\{v+t L(v): v \in$ $V\}$. It is easy to check that each $\widetilde{V}_{t}$ is an $n$-dimensional space with $\widetilde{V}_{t} \cap W=\{0\}$. Applying Lemma 2.4 to a basis $v_{1}, \ldots, v_{k}$ of $V$ and $w_{i}:=v_{i}+t L\left(v_{i}\right)$, it is easy to see that $\gamma\left(V, \widetilde{V}_{t}\right) \rightarrow 0(t \rightarrow 0+)$, which implies our assertion.

Lemma 2.7. Let $X$ be a Banach space, $1 \leq n<\operatorname{dim} X$, and $K \geq 1$. Let $X=$ $E \oplus F$, where $F$ is an n-dimensional space. Suppose that the canonical mapping $\mu: E \oplus F \rightarrow E \times F$ (where $E \times F$ is equipped with the maximum norm) is $K$ bilipschitz. Then there exists $\omega>0$ such that if $\widetilde{F} \subset X$ is a closed space with $\gamma(F, \widetilde{F})<\omega$, then $X=E \oplus \widetilde{F}$ and the canonical mapping $\widetilde{\mu}: E \oplus \widetilde{F} \rightarrow E \times \widetilde{F}$ is $2 K$-bilipschitz.

Proof. Distinguishing the cases $\lambda<1, \lambda=1$ and $\lambda>1$, it is easy to check that there exists $1>\omega_{0}>0$ such the inequalities

$$
\begin{aligned}
& K \max (1+\omega, \lambda)+\omega \leq 2 K \max (1, \lambda), \\
& \quad K^{-1} \max (1-\omega, \lambda)-\omega \geq(2 K)^{-1} \max (1, \lambda)
\end{aligned}
$$

hold for each $\lambda \geq 0$ and $0<\omega<\omega_{0}$. By Lemma 2.3(iii), we can choose $0<\omega<\omega_{0}$ such that $X=E \oplus \widetilde{F}$ whenever $\gamma(F, \widetilde{F})<\omega$. Let $\widetilde{F}$ with $\gamma(F, \widetilde{F})<\omega$ be given, and consider arbitrary $\widetilde{f} \in \widetilde{F}$ and $e \in E$. We will prove

$$
(2 K)^{-1} \max (\|\widetilde{f}\|,\|e\|) \leq\|\widetilde{f}+e\| \leq 2 K \max (\|\widetilde{f}\|,\|e\|) .
$$


Since the case $\tilde{f}=0$ is trivial, by homogenuity of the norm we can suppose $\|\widetilde{f}\|=1$ and find $f \in F$ with $\|f-\widetilde{f}\|<\omega$. Applying (2.3) to $\lambda:=\|e\|$, we obtain

$$
\begin{aligned}
\|\tilde{f}+e\| \leq\|f+e\|+\omega \leq K \max (\|f\|,\|e\|)+\omega & \\
& \leq K \max (1+\omega,\|e\|)+\omega \leq 2 K \max (1,\|e\|)
\end{aligned}
$$

and

$$
\|\tilde{f}+e\| \geq\|f+e\|-\omega \geq K^{-1} \max (1-\omega,\|e\|)-\omega \geq(2 K)^{-1} \max (1,\|e\|) .
$$

Thus, (2.4) holds, and $\widetilde{\mu}$ is (2K)-bilipschitz.

\section{Properties of Lipschitz SURfaces of Finite CODIMENSION}

If $X$ is a Banach space and $X=E \oplus F$, then we denote by $\pi_{E, F}$ the projection of $X$ on $E$ along the space $F$.

Definition 3.1. Let $X$ be a Banach space and $A \subset X$.

(i) Let $F$ be a closed subspace of $X$. We say that $A$ is an $F$-Lipschitz surface if there exists a topological complement $E$ of $F$ and a Lipschitz mapping $\varphi: E \rightarrow F$ such that $A=\{x+\varphi(x): x \in E\}$.

(ii) Let $1 \leq n<\operatorname{dim} X$ be a natural number. We say that $A$ is a Lipschitz surface of codimension $n$ if $A$ is an $F$-Lipschitz surface for some $n$-dimensional space $F \subset X$.

(iii) If we consider in (i) mappings $\varphi: E \rightarrow F$ which are d.c. (resp. Lipschitz d.c., locally d.c., Lipschitz locally d.c.), we obtain the notions of an $F$-d.c. surface, d.c. surface of codimension $n$ (resp. $F$-Lipschitz d.c. surface, etc.). A Lipschitz surface (resp. d.c. surface, etc.) of codimension 1 is said to be a Lipschitz hypersurface (resp. d.c. hypersurface, etc.).

(iv) The $\sigma$-ideals of sets which can be covered by countably many Lipschitz surfaces (d.c. surfaces) of codimension $n$ will be denoted by $\mathcal{L}^{n}(X)\left(\mathcal{D C} \mathcal{C}^{n}(X)\right)$, respectively.

Lemma 3.2. Let $X$ be a Banach space, $F \subset X$ a space of dimension $n(1 \leq n<$ $\operatorname{dim} X$ ), and $A \subset X$. Then the following properties are equivalent.

(i) $A$ is an F-Lipschitz surface (resp. an F-d.c. surface, an F-Lipschitz d.c. surface, an F-Lipschitz locally d.c. surface).

(ii) There exists a topological complement $\widetilde{E}$ of $F$ such that $\left.\widetilde{\pi}\right|_{A}: A \rightarrow \widetilde{E}$ is a bijection and $\left(\left.\widetilde{\pi}\right|_{A}\right)^{-1}$ is Lipschitz (resp. d.c., etc.), where $\widetilde{\pi}:=\pi_{\widetilde{E}, F}$.

(iii) If $X=F \oplus E$ and $\pi:=\pi_{E, F}$, then $\left.\pi\right|_{A}: A \rightarrow E$ is a bijection and $\left(\left.\pi\right|_{A}\right)^{-1}$ is Lipschitz (resp. d.c., etc.).

(iv) If $X=F \oplus E$, then there exists a Lipschitz mapping (resp. a d.c. mapping, etc.) $\varphi: E \rightarrow F$ such that $A=\{x+\varphi(x): x \in E\}$.

Proof. In the proof we use Lemma 2.2(i)-(iii).

If (i) holds, then there exists a topological complement $\widetilde{E}$ of $F$ and a Lipschitz (d.c., etc.) mapping $\widetilde{\varphi}: \widetilde{E} \rightarrow F$ such that $A=\{x+\widetilde{\varphi}(x): x \in \widetilde{E}\}$. Set $\widetilde{\pi}:=\pi_{\widetilde{E}, F}$. Then clearly $\left.\widetilde{\pi}\right|_{A}: A \rightarrow \widetilde{E}$ is a bijection and $\left(\left.\widetilde{\pi}\right|_{A}\right)^{-1}$ is Lipschitz (d.c., etc.), since $\left(\left.\widetilde{\pi}\right|_{A}\right)^{-1}(\widetilde{e})=\widetilde{e}+\widetilde{\varphi}(\widetilde{e})$. 
Now let $\widetilde{E}$ be as in (ii), and let $E$ and $\pi$ be as in (iii). Since $\left.\pi\right|_{\widetilde{E}}: \widetilde{E} \rightarrow E$ is clearly a linear isomorphism, $\left(\left.\pi\right|_{\widetilde{E}}\right)^{-1}=\left.\widetilde{\pi}\right|_{E},\left.\pi\right|_{A}=\left(\left.\pi\right|_{\widetilde{E}}\right) \circ\left(\left.\widetilde{\pi}\right|_{A}\right)$ and $\left(\left.\pi\right|_{A}\right)^{-1}=$ $\left(\left.\widetilde{\pi}\right|_{A}\right)^{-1} \circ\left(\left.\widetilde{\pi}\right|_{E}\right)$, we easily obtain (iii).

Letting $\varphi(x):=\left(\left.\pi\right|_{A}\right)^{-1}(x)-x$ for $x \in E$, we easily see that (iii) implies (iv). The implication (iv) $\Rightarrow$ (i) is trivial.

Remark 3.3. $\quad$ (i) Every Lipschitz surface of codimension $n$ in $X$ is clearly a closed subset of $X$.

(ii) If $S \subset X$ is a Lipschitz (resp. d.c., etc.) surface of codimension $n \geq 2$, then $S$ is a subset of a Lipschitz (resp. d.c., etc.) surface of codimension $n-1$. Indeed, suppose that $S=\{x+\varphi(x): x \in E\}$, where $\varphi: E \rightarrow F, X=E \oplus F$, and $F$ is $n$-dimensional. Choose $0 \neq v \in F$, and write $F=\operatorname{sp}\{v\} \oplus \widetilde{F}$. Set $\widetilde{E}:=E+\operatorname{sp}\{v\}$ and, for $x \in \widetilde{E}$, define $\widetilde{\varphi}(x):=\pi_{\widetilde{F}, \widetilde{E}}\left(\varphi\left(\pi_{E, F}(x)\right)\right)$. Set $\widetilde{S}:=\{y+\widetilde{\varphi}(y): y \in \widetilde{E}\}$. It is easy to see that $S \subset \widetilde{S}$ and $\widetilde{\varphi}: \widetilde{E} \rightarrow F$ is Lipschitz (resp. d.c., etc) if $\varphi$ is Lipschitz (resp. d.c., etc.).

Consequently, if $\operatorname{dim} X>n \geq 2$, then $\mathcal{L}^{n}(X) \subset \mathcal{L}^{n-1}(X)$. If $X$ is separable, then this inclusion is proper, see Remark 4.8, which shows that no Lipschitz surface of codimension $n-1$ belongs to $\mathcal{L}^{n}(X)$ (if $\operatorname{dim} X<$ $\infty$, it is sufficient to use by the obvious way basic properties of Hausdorff dimension).

(iii) If $X$ is separable, then the $\sigma$-ideal $\mathcal{D} \mathcal{C}^{n}(X)$ coincides with the $\sigma$-ideal generated by Lipschitz d.c. surfaces (or Lipschitz locally d.c. surfaces, or locally d.c. surfaces). It easily follows from local Lipschitzness of d.c. functions, from the well-known fact that each Lipschitz convex function defined an an open ball in a space $E$ can be extended to a Lipschitz convex function on $E$, and from separability of $X$.

(iv) It is not difficult to show that $\mathcal{D C}^{n}(X)$ is a proper subset of $\mathcal{L}^{n}(X)$ (if $\operatorname{dim} X>n \geq 1)$; see [17, p. 295] for $n=1$.

Remark 3.4. Suppose that $X=E \oplus F$ and $F$ is finite dimensional. An easy argument using local compactness of $F$ shows that $\pi_{E, F}(A)$ is closed in $E$ whenever $A$ is closed and bounded in $X$. Consequently, $\pi_{E, F}(A)$ is an $F_{\sigma}$ subset of $E$ whenever $A$ is closed in $X$.

We will need the following well-known easy consequence of the Brouwer's Invariance of Domain Theorem. Because of the lack of a suitable reference, we present a short proof.

Lemma 3.5. Let $C, \widetilde{C}$ be Banach spaces with $0<\operatorname{dim} C=\operatorname{dim} \widetilde{C}<\infty$ and let $f: \widetilde{C} \rightarrow C$ be an injective continuous mapping such that $f^{-1}: f(\widetilde{C}) \rightarrow \widetilde{C}$ is Lipschitz. Then $f(\widetilde{C})=C$.

Proof. We can clearly suppose that $C=\widetilde{C}$ and $X:=C=\widetilde{C}$ is an Euclidean space. The Brouwer's Invariance of Domain Theorem implies that $f(X)$ is open in $X$. Let $y_{n} \rightarrow y$, where $y_{n} \in f(X)$. Then $\left(y_{n}\right)$ is bounded and, since $f^{-1}$ is Lipschitz, $\left(x_{n}\right):=\left(f^{-1}\left(y_{n}\right)\right)$ is bounded as well. Choose a subsequence $x_{n_{k}} \rightarrow x \in X$. Then $f\left(x_{n_{k}}\right)=y_{n_{k}} \rightarrow f(x)=y$. Thus, we have proved that $f(X)$ is closed; the connectivity of $X$ implies $f(X)=X$.

Proposition 3.6. Let $X$ be a Banach space, $S \subset X$ a Lipschitz surface of codimension $n$, and let $X=D \oplus F$ with $\operatorname{dim} F=n$. Let $\psi=\left.\pi_{D, F}\right|_{S}: S \rightarrow D$ be injective 
and $\psi^{-1}: \psi(S) \rightarrow S$ be Lipschitz. Then $S$ is an F-Lipschitz surface. Moreover, if $S$ is a Lipschitz locally d.c. surface of codimension n, then $S$ is an F-Lipschitz locally d.c. surface.

Proof. Choose an $n$-dimensional space $\widetilde{F}$ such that $S$ is an $\widetilde{F}$-Lipschitz surface. Since the case $F=\widetilde{F}$ is obvious by Lemma 3.2 we suppose $F \neq \widetilde{F}$. Put $K:=F \cap \widetilde{F}$ and choose spaces $C, \widetilde{C}$ such that $F=K \oplus \widetilde{C}$ and $\widetilde{F}=K \oplus C$. Then clearly $1 \leq \operatorname{dim} C=\operatorname{dim} \widetilde{C}<\infty$. Choose a topological complement $Z$ of the (finite dimensional) space $F+\widetilde{F}=K \oplus C \oplus \widetilde{C}$ and denote $E:=Z \oplus C, \widetilde{E}:=Z \oplus \widetilde{C}$. Clearly $X=F \oplus E=\widetilde{F} \oplus \widetilde{E}$.

By Lemma 3.2, $\widetilde{\varphi}:=\left.\pi_{\widetilde{E}, \widetilde{F}}\right|_{S}: S \rightarrow \widetilde{E}$ is a bilipschitz bijection. It is easy to see (proceeding similarly as in the proof of Lemma 3.2) that $\varphi:=\left.\pi_{E, F}\right|_{S}: S \rightarrow E$ is injective and $\varphi^{-1}: \varphi(S) \rightarrow S$ is Lipschitz. So Lemma 3.2 implies that, to prove the first part of the assertion, it is sufficient to verify $\varphi(S)=E$.

To this end choose an arbitrary $e \in E$ and write $e=z+c$, where $z \in Z$ and $c \in C$. For each $x \in \widetilde{C}$, put $f(x):=\varphi \circ(\widetilde{\varphi})^{-1}(x+z)-z$. Clearly $f(x) \in$ $(F+\widetilde{F}) \cap E=C$; so $f: \widetilde{C} \rightarrow C$. It is easy to see that $f$ is continuous injective and $f^{-1}(y)=\widetilde{\varphi} \circ \varphi^{-1}(y+z)-z$ for each $y \in f(\widetilde{C})$. Consequently, $f^{-1}$ is Lipschitz, and so $f(\widetilde{C})=C$ by Lemma 3.5. For $\widetilde{c}:=f^{-1}(c)$ we have $\varphi\left((\widetilde{\varphi})^{-1}(\widetilde{c}+z)\right)=c+z=e$; so $\varphi(S)=E$.

To prove the second part of the assertion, we suppose that $(\widetilde{\varphi})^{-1}: \widetilde{E} \rightarrow X$ is moreover locally d.c. Then $g:=\varphi \circ(\widetilde{\varphi})^{-1}=\pi_{E, F} \circ(\widetilde{\varphi})^{-1}$ is clearly Lipschitz and it is locally d.c. by Lemma 2.2 (i). Since $\varphi(S)=E$, we have that $g: \widetilde{E} \rightarrow E$ is a bijection and $g^{-1}=\widetilde{\varphi} \circ \varphi^{-1}$ is Lipschitz. Choose a linear bijection $L: \widetilde{C} \rightarrow C$, and let $G: \widetilde{E} \rightarrow E$ be the mapping which assigns to a point $\widetilde{e}=\widetilde{c}+z(\widetilde{c} \in \widetilde{C}$, $z \in Z)$ the point $G(\widetilde{e}):=L(\widetilde{c})+z$. Then clearly $G$ is a linear isomorphism. Since $G(\widetilde{e})-\widetilde{e} \in C+\widetilde{C}$ and $g(\widetilde{e})-\widetilde{e} \in F+\widetilde{F}$, we obtain that $g-G$ has a finite dimensional range. Consequently, Lemma 2.2(v) implies that $g^{-1}$ is locally d.c. Thus, Lemma 2.2 (iv) implies that $\varphi^{-1}=(\widetilde{\varphi})^{-1} \circ g^{-1}$ is locally d.c. So, Lemma 3.2 implies that $S$ is an $F$-Lipschitz locally d.c. surface.

Proposition 3.7. Let $X$ be a Banach space, $F \subset X$ an $n$-dimensional space, and $A \subset X$ an F-Lipschitz (resp. F-Lipschitz locally d.c.) surface. Then there exists $\varepsilon>0$ such that if $\widetilde{F} \subset X$ is an $n$-dimensional space with $\gamma(F, \widetilde{F})<\varepsilon$, then $A$ is an $\widetilde{F}$-Lipschitz (resp. $\widetilde{F}$-Lipschitz locally d.c.) surface.

Proof. Choose $E$ such that $X=E \oplus F$, and choose $K \geq 1$ such that the canonical mapping $\gamma: E \oplus F \rightarrow E \times F$ is $K$-bilipschitz. Choose a corresponding $\omega>0$ by Lemma 2.7. Denote $\pi:=\pi_{E, F}$, and choose $L \geq 1$ such that $\left(\left.\pi\right|_{A}\right)^{-1}$ is Lipschitz with constant $L$. Choose $\varepsilon>0$ such that $\varepsilon<\omega$ and

$$
2 K^{2} L \varepsilon<1 / 2 \text {. }
$$

Now suppose that an $n$-dimensional space $\widetilde{F}$ with $\gamma(F, \widetilde{F})<\varepsilon$ be given. Since $\varepsilon<\omega$, we have that $X=E \oplus \widetilde{F}$ and the canonical mapping $\widetilde{\gamma}: E \oplus \widetilde{F} \rightarrow E \times \widetilde{F}$ is $2 K$-bilipschitz. By Proposition 3.6 it is sufficient to prove that, putting $\widetilde{\pi}:=\pi_{E, \widetilde{F}}$, the mapping $\left(\left.\widetilde{\pi}\right|_{A}\right)^{-1}$ is Lipschitz with constant $2 L$; i.e., that

$$
\|x-y\| \leq 2 L\|\widetilde{\pi}(x)-\widetilde{\pi}(y)\|=2 L\|\widetilde{\pi}(x-y)\|, \quad x, y \in A .
$$


Thus, consider $x, y \in A, x \neq y$, and write $x-y=e_{1}+f=e_{2}+\tilde{f}$, where $e_{1}=\pi(x-y) \in E, e_{2}=\widetilde{\pi}(x-y) \in E, f \in F$, and $\widetilde{f} \in \widetilde{F}$. We know that $\|x-y\| \leq L\left\|e_{1}\right\|$ and so $\|\tilde{f}\| \leq 2 K\|x-y\| \leq 2 K L\left\|e_{1}\right\|$.

If $\widetilde{f}=0$, then (3.2) is obvious. If $\tilde{f} \neq 0$, put $\widetilde{z}:=\|\tilde{f}\|^{-1} \tilde{f}$, and find $z \in F$ such that $\|\widetilde{z}-z\| \leq \varepsilon$. Then $f_{2}:=\|\widetilde{f}\| z$ satisfies $\left\|\widetilde{f}-f_{2}\right\| \leq \varepsilon\|\widetilde{f}\|$, and so

$$
K^{-1}\left\|e_{1}-e_{2}\right\| \leq\left\|e_{1}-e_{2}+f-f_{2}\right\|=\left\|\widetilde{f}-f_{2}\right\| \leq \varepsilon\|\widetilde{f}\| \leq 2 K L \varepsilon\left\|e_{1}\right\| .
$$

Thus, by (3.1), we obtain $\left\|e_{1}-e_{2}\right\| \leq\left\|e_{1}\right\| / 2$, and so $\left\|e_{2}\right\| \geq\left\|e_{1}\right\| / 2$. Therefore $\|x-y\| \leq L\left\|e_{1}\right\| \leq 2 L\left\|e_{2}\right\|$, which proves (3.2) and finishes the proof.

Remark 3.8. I do not know whether the analogies of Proposition 3.6 and Proposition 3.7 hold for Lipschitz d.c. surfaces.

\section{Projections of Lipschitz Surfaces of Finite CODimension}

Definition 4.1. Let $X$ be a separable Banach space, and let a finite-dimensional space $V \subset X$ be given. We define the following classes of sets:

(i) $\mathcal{A}(V)$ is the system of all Borel sets $B \subset X$ such that $V \cap(B+a)$ is Lebesgue null (in $V$ ) for each $a \in X$. For $0 \neq v \in X$, we put $\mathcal{A}(v):=\mathcal{A}(\operatorname{sp}\{v\})$.

(ii) $\mathcal{A}^{*}(V, \varepsilon)$ (where $0<\varepsilon<1$ ) is the system of all Borel sets $B \subset X$ such that $B \in \mathcal{A}(W)$ for each space $W$ with $\gamma(V, W)<\varepsilon$, and $\mathcal{A}^{*}(V)$ is the system of all sets $B$ such that $B=\bigcup_{k=1}^{\infty} B_{k}$, where $B_{k} \in \mathcal{A}^{*}\left(V, \varepsilon_{k}\right)$ for some $0<\varepsilon_{k}<1$.

(iii) $\mathcal{C}_{d}^{*}$ (where $d \in \mathbf{N}$ ) is the system of those $B \subset X$ that can be written as $B=\bigcup_{k=1}^{\infty} B_{k}$, where each $B_{k}$ belongs to $\mathcal{A}^{*}\left(V_{k}\right)$ for some $V_{k}$ with $\operatorname{dim} V_{k}=d$.

(iv) $\mathcal{A}$ is the system of those $B \subset X$ that can be, for every complete sequence $\left(v_{k}\right)$ in $X$, written as $B=\bigcup_{k=1}^{\infty} B_{k}$, where each $B_{k}$ belongs to $\mathcal{A}\left(v_{k}\right)$.

Note that $\mathcal{C}_{1}^{*}$ coincide with $\mathcal{C}^{*}$ from [14] and $\mathcal{A}$ is the system of all Aronszajn null sets. For basic properties of sets from $\mathcal{A}$ see [2]. Lemma 2.5] easily implies the following fact.

Lemma 4.2. Let $X, Y$ be Banach spaces and $F: X \rightarrow Y$ a linear isomorphism. Let $S \subset X$ belong to $\mathcal{A}^{*}(V, \delta)$. Then there exists $\varepsilon>0$ such that $F(S) \in \mathcal{A}^{*}(F(V), \varepsilon)$ (in the space $Y$ ).

Proposition 4.3. Let $X$ be a separable infinite dimensional Banach space and $k \in \mathbf{N}$. Then $\mathcal{C}_{1}^{*} \subset \mathcal{C}_{2}^{*} \subset \cdots \subset \mathcal{A}$ and all inclusions are proper.

Proof. To prove the inclusions $\mathcal{C}_{d}^{*} \subset \mathcal{A}$, it is sufficient to show that $\mathcal{A}^{*}(V, \varepsilon) \subset \mathcal{A}$ whenever $V \subset X$ is a $d$-dimensional space and $\varepsilon>0$. Let $V, \varepsilon$ and $B \in \mathcal{A}^{*}(V, \varepsilon)$ be given. Choose a basis $\left(v_{1}, \ldots, v_{d}\right)$ of $V$ and consider an arbitrary complete sequence $\left(u_{i}\right)$ in $X$. Choose a $\delta>0$ that corresponds to $\left(v_{1}, \ldots, v_{d}\right)$ and $\varepsilon$ by Lemma 2.4. We can clearly choose $n \in \mathbf{N}$ and vectors $w_{1}, \ldots w_{d}$ in $U:=\operatorname{sp}\left\{u_{1}, \ldots, u_{n}\right\}$ such that $\left\|w_{i}-v_{i}\right\|<\delta, i=1, \ldots, d$. Then, denoting $W:=\operatorname{sp}\left\{w_{1}, \ldots, w_{d}\right\}$, we have $\gamma(V, W)<\varepsilon$, and so $B \in \mathcal{A}(W)$. Consequently, by the Fubini theorem, $B \in \mathcal{A}(U)$. Using [2, Proposition 6.29], we easily obtain that $B$ can be decomposed as $B=\bigcup_{i=1}^{n} B_{i}$, where $B_{i} \in \mathcal{A}\left(u_{i}\right)$. So, $B \in \mathcal{A}$, and $\mathcal{C}_{d}^{*} \subset \mathcal{A}$ is proved.

To prove $\mathcal{C}_{d}^{*} \subset \mathcal{C}_{d+1}^{*}$, consider a $B \in \mathcal{A}^{*}(V, \varepsilon)$, where $\operatorname{dim} V=d$ and $1>\varepsilon>0$. Choose a basis $v_{1}, \ldots, v_{d}$ of $V$ with $\left\|v_{i}\right\|=1$ and find a corresponding $\delta>0$ by 
Lemma 2.4. Now choose an arbitrary $Z \supset V$ with $\operatorname{dim} Z=d+1$. To prove $B \in \mathcal{A}^{*}(Z, \delta)$, consider an arbitrary $(d+1)$-dimensional $W$ with $\gamma(W, Z)<\delta$. By the definition of $\gamma$, find $w_{1}, \ldots, w_{d} \in W$ with $\left\|w_{1}-v_{1}\right\|<\delta, \ldots,\left\|w_{d}-v_{d}\right\|<\delta$, and set $\widetilde{W}:=\operatorname{sp}\left\{w_{1}, \ldots, w_{d}\right\}$. The choice of $\delta$ implies that $\gamma(\widetilde{W}, V)<\varepsilon$, and so $\widetilde{W} \cap(B+a)$ is Lebesgue null in $\widetilde{W}$ for each $a \in X$. Consequently, by Fubini theorem, $W \cap(B+a)$ is Lebesgue null in $W$ for each $a \in X$. So $B \in \mathcal{A}^{*}(Z, \delta)$, and $\mathcal{C}_{d}^{*} \subset \mathcal{C}_{d+1}^{*}$ follows.

A construction of a set in $\mathcal{A} \backslash \mathcal{C}_{1}^{*}$ is presented in the proof of [14, Proposition 13]. Moreover, it is shown in [14 that this set $\left(F_{2}(I)\right)$ meets any 2-dimensional affine space in a 2-dimensional Lebesgue null set, which shows that even $\mathcal{C}_{2}^{*} \backslash \mathcal{C}_{1}^{*} \neq \emptyset$. It is not difficult to modify that construction and obtain a set in $\mathcal{C}_{d+1}^{*} \backslash \mathcal{C}_{d}^{*}$ for each $d$ (see Remark 4.4). However, since the notation is somewhat complicated in the general case, we will give a detailed proof for $d=2$ only.

Our construction starts quite similarly as the construction of a set from $\mathcal{A} \backslash$ $\mathcal{C}^{*}$ on p. 20 of [14. Namely, by the same procedure as in 14 we can define positive numbers $c_{0}, c_{1}, c_{2}, \ldots$ and nonzero vectors $u_{0}, u_{1}, u_{2}, \ldots$ in $X$ such that both $\left\{u_{6 n-3}: n \in \mathbf{N}\right\}$ and $\left\{u_{6 n}: n \in \mathbf{N}\right\}$ are dense in $X$, and the formula $F(x)=\sum_{k=0}^{\infty} c_{k} x_{k+1} u_{k}$ (where $x=\left(x_{1}, x_{2}, \ldots\right)$ ) defines a linear injective mapping of $\ell_{\infty}$ to $X$.

As in [14, we set $I:=\left\{x \in \ell_{\infty}: 1 \leq x_{k} \leq 2\right\}$, and equip $I$ with the topology of pointwise convergence (so it is a compact metrizable space) and with the measure $\mu$ defined as the product of countably many copies of the Lebesgue measure on $[1,2]$.

Choose two sequences $\xi_{1}^{1}, \xi_{2}^{1}, \ldots$ and $\xi_{1}^{2}, \xi_{2}^{2}, \ldots$ such that $0<\xi_{j}^{1}<1 /(j+1)$ !, $0<\xi_{j}^{2}<1 /(j+1)$ !, and

$$
\lim _{k \rightarrow \infty} \sum_{j=k}^{\infty} c_{3 j-2} \xi_{j}^{1} 2^{j}\left\|u_{3 j-2}\right\| / c_{6 k-3}=0, \quad \lim _{k \rightarrow \infty} \sum_{j=k}^{\infty} c_{3 j-1} \xi_{j}^{2} 2^{j}\left\|u_{3 j-1}\right\| / c_{6 k}=0 .
$$

Now, for $x \in I$, set

$G(x)=\sum_{k=1}^{\infty} c_{3 k-2} \xi_{k}^{1} x_{1} x_{3} \ldots x_{2 k-1} u_{3 k-2}+\sum_{k=1}^{\infty} c_{3 k-1} \xi_{k}^{2} x_{2} x_{4} \ldots x_{2 k} u_{3 k-1}+\sum_{k=1}^{\infty} x_{k} c_{3 k} u_{3 k}$.

Then $G: I \rightarrow X$ is a continuous mapping. Indeed, we have $G=F \circ H$, where

$H\left(x_{1}, x_{2}, \ldots\right):=\left(0, \xi_{1}^{1} x_{1}, \xi_{1}^{2} x_{2}, x_{1}, \xi_{2}^{1} x_{1} x_{3}, \xi_{2}^{2} x_{2} x_{4}, x_{2}, \xi_{3}^{1} x_{1} x_{3} x_{5}, \xi_{3}^{2} x_{2} x_{4} x_{6}, x_{3}, \ldots\right)$,

and $H: I \rightarrow \ell_{\infty}$ is clearly continuous. So, $G(I)$ is compact.

Let $e_{j}$ be the $j$-th member of the canonical basis of $\ell_{\infty}$. Observe that if $x \in I$, $k_{1}, k_{2} \in \mathbf{N}, t, \tau \in \mathbf{R}$ and $x+t e_{2 k_{1}-1}+\tau e_{2 k_{2}} \in I$, then $G\left(x+t e_{2 k_{1}-1}+\tau e_{2 k_{2}}\right)=$ $G(x)+t v_{k_{1}}(x)+\tau w_{k_{2}}(x)$, where

$$
\begin{gathered}
v_{k}(x):=\sum_{j=k}^{\infty} c_{3 j-2} \xi_{j}^{1}\left(x_{1} x_{3} \ldots x_{2 j-1} / x_{2 k-1}\right) u_{3 j-2}+c_{6 k-3} u_{6 k-3}, \\
w_{k}(x):=\sum_{j=k}^{\infty} c_{3 j-1} \xi_{j}^{2}\left(x_{2} x_{4} \ldots x_{2 j} / x_{2 k}\right) u_{3 j-1}+c_{6 k} u_{6 k} .
\end{gathered}
$$

Now consider $x, y \in I$ such that $x \neq y$ and $t G(x)+(1-t) G(y) \in G(I)$ for infinitely many real $t$. Since $F$ is a linear injection of $\ell_{\infty}$ to $X$, for any such $t$ we have $t H(x)+(1-t) H(y)=H(z)$ for some $z \in I$. Considering $(3 k+1)$-th coordinates 
of $H(z)$ we obtain $z=t x+(1-t) y$. Consequently, considering $(3 k-1)$-th and $3 k$-th coordinates of $H(z)$, we obtain that, for each $k \in \mathbf{N}$,

$$
\begin{aligned}
& t x_{1} x_{3} \ldots x_{2 k-1}+(1-t) y_{1} y_{3} \ldots y_{2 k-1}=\left(t x_{1}+(1-t) y_{1}\right) \cdots\left(t x_{2 k-1}+(1-t) y_{2 k-1}\right), \\
& t x_{2} x_{4} \ldots x_{2 k}+(1-t) y_{2} y_{4} \ldots y_{2 k}=\left(t x_{2}+(1-t) y_{2}\right) \cdots\left(t x_{2 k}+(1-t) y_{2 k}\right) .
\end{aligned}
$$

Since the above equalities hold for infinitely many $t$, we infer that $x$ and $y$ differ at most in one odd coordinate and at most in one even coordinate (otherwise one of right sides, for sufiicintly large $k$, is a polynomial in $t$ of degree grater than one, which is impossible). Consequently, there exist $k_{1}, k_{2} \in \mathbf{N}$ such that $y \in x+\operatorname{sp}\left\{e_{2 k_{1}-1}, e_{2 k_{2}}\right\} ;$ so $G(y) \in G(x)+\operatorname{sp}\left\{v_{k_{1}}(x), w_{k_{2}}(x)\right\}$.

The above analysis shows that the set of lines, which contain any fixed point $G(x), x \in I$, and meet the set $G(I)$ in an infinite set, can be covered by countably many planes containing $G(x)$. Therefore $G(I)$ meets any 3-dimensional affine subspace of $X$ in a set of three dimensional Lebesgue measure zero. Consequently, $G(I) \in \mathcal{C}_{3}^{*}$.

Now suppose that $G(I) \in \mathcal{C}_{2}^{*}$, hence $G(I)=\bigcup_{n=1}^{\infty} B_{n}$, where $B_{n} \in \mathcal{A}^{*}\left(V_{n}, \varepsilon_{n}\right)$ and $V_{n}$ are two-dimensional subspaces of $X$. Write $V_{n}=\operatorname{sp}\left\{p_{n}, q_{n}\right\}$ and choose $\delta_{n}>0$ (by Lemma 2.4) such that $\gamma\left(V_{n}, \operatorname{sp}\{v, w\}\right)<\varepsilon_{n}$ whenever $\left\|v-p_{n}\right\|<\delta_{n}$ and $\left\|w-q_{n}\right\|<\delta_{n}$. For any given $n$ find $k_{1}, k_{2}$ such that

$$
\begin{gathered}
\sum_{j=k_{1}}^{\infty} 2^{j} c_{3 j-2} \xi_{j}^{1}\left\|u_{3 j-2}\right\|<c_{6 k_{1}-3} \delta_{n} / 2, \quad\left\|u_{6 k_{1}-3}-p_{n}\right\|<\delta_{n} / 2, \\
\sum_{j=k_{2}}^{\infty} 2^{j} c_{3 j-1} \xi_{j}^{2}\left\|u_{3 j-1}\right\|<c_{6 k_{2}} \delta_{n} / 2, \quad\left\|u_{6 k_{2}}-q_{n}\right\|<\delta_{n} / 2 .
\end{gathered}
$$

For any $x \in I$ we have

$$
\begin{gathered}
\left\|v_{k_{1}}(x)-c_{6 k_{1}-3} p_{n}\right\| \leq \sum_{j=k_{1}}^{\infty} 2^{j} c_{3 j-2} \xi_{j}^{1}\left\|u_{3 j-2}\right\|+c_{6 k_{1}-3}\left\|u_{6 k_{1}-3}-p_{n}\right\|<c_{6 k_{1}-3} \delta_{n}, \\
\left\|w_{k_{2}}(x)-c_{6 k_{2}} q_{n}\right\| \leq \sum_{j=k_{2}}^{\infty} 2^{j} c_{3 j-1} \xi_{j}^{1}\left\|u_{3 j-1}\right\|+c_{6 k_{2}}\left\|u_{6 k_{2}}-q_{n}\right\|<c_{6 k_{2}} \delta_{n} .
\end{gathered}
$$

So $\left\|v_{k_{1}}(x) / c_{6 k_{1}-3}-p_{n}\right\|<\delta_{n}$ and $\left\|w_{k_{2}}(x) / c_{6 k_{2}}-q_{n}\right\|<\delta_{n}$, which shows that the plane $G(x)+\operatorname{sp}\left\{v_{k_{1}}(x), w_{k_{2}}(x)\right\}$ meets $B_{n}$ in a two-dimensional Lebesgue null set. Hence the set

$\left\{(t, \tau): x+t e_{2 k_{1}-1}+\tau e_{2 k_{2}} \in G^{-1}\left(B_{n}\right)\right\}=\left\{(t, \tau): G(x)+t v_{k_{1}}(x)+\tau w_{k_{2}}(x) \in B_{n}\right\}$ is Lebesgue null, and Fubini theorem gives $\mu\left(G^{-1}\left(B_{n}\right)\right)=0$. (Note that $G^{-1}\left(B_{n}\right)$ is Borel, since $G$ is continuous.) But this contradicts $I=\bigcup_{n=1}^{\infty} G^{-1}\left(B_{n}\right)$, and we infer that $G(I) \notin \mathcal{C}_{2}^{*}$.

Remark 4.4. For an arbitrary $d \in \mathbf{N}$, we obtain as above that $G_{d}(I) \in \mathcal{C}_{d+1}^{*} \backslash \mathcal{C}_{d}^{*}$, where $G_{d}=F \circ H_{d}$,

$$
H_{d}(x):=\left(0, \xi_{1}^{1} x_{1}, \ldots, \xi_{1}^{d} x_{d}, x_{1}, \xi_{2}^{1} x_{1} x_{d+1}, \ldots, \xi_{2}^{d} x_{d} x_{2 d}, x_{2}, \xi_{3}^{1} x_{1} x_{d+1} x_{2 d+1}, \ldots\right),
$$

and $\left(\xi_{i}^{1}\right), \ldots,\left(\xi_{i}^{d}\right)$ are suitably chosen sequences. 
Proposition 4.5. Let $X$ be a separable infinite dimensional space, $S$ a Lipschitz surface of codimension $n \geq 2$, and $P: X \rightarrow Y$ a continuous linear mapping onto a Banach space $Y$ such that $\operatorname{dim}(\operatorname{ker}(P))<n$. Then there exists an $n$-dimensional space $D \subset Y$ and $0<\varepsilon<1$ such that $P(S) \in \mathcal{C}^{*}(D, \varepsilon)$ in $Y$. Consequently, $P(S)$ is a first category subset of $Y$ which is Aronszajn null in $Y$.

Proof. Denote $K:=\operatorname{ker} P$. Choose a space $F \subset X$ such that $\operatorname{dim} F=n$ and $S$ is an $F$-Lipschitz surface. Using Lemma 3.7. Lemma 2.3 and Lemma 2.6, we can choose a space $V$ with $\operatorname{dim} V=n$ such that $S$ is an $V$-Lipschitz surface and $V \cap K=\{0\}$. Choose a closed space $H \subset X$ such that $X=H \oplus(K \oplus V)$. Denoting $Z:=H \oplus V$, we have $X=Z \oplus K$. Set $\pi:=\pi_{Z, K}$. Using Lemma 3.7 and Lemma 2.3, we find $0<\delta<1$ such that $\gamma(V, W)<\delta$ implies that $S$ is a $W$-Lipschitz surface and $W \oplus(H \oplus K)=X$. Now consider on arbitrary $W \subset Z$ such that $\gamma(V, W)<\delta$. We can choose a Lipschitz mapping $\varphi: H \oplus K \rightarrow W$ such that $S=\{h+k+\varphi(h+k)$ : $h \in H, k \in K\}$. Consequently, $\pi(S)=\{h+\varphi(h+k): h \in H, k \in K\}$. Now consider an arbitrary $a=h_{0}+w_{0} \in Z$. Then $(\pi(S)+a) \cap W=\left\{w_{0}+\varphi\left(-h_{0}+k\right): k \in K\right\}$. Since the mapping $\psi: K \rightarrow W$ defined by $\psi(k):=w_{0}+\varphi\left(-h_{0}+k\right)$ is Lipschitz and $\operatorname{dim} K<\operatorname{dim} W$, we obtain that $(\pi(S)+a) \cap W$ is Lebesgue null in $W$. Since $\pi(S)$ is an $F_{\sigma}$ set by Remark 3.3(i) and Remark 3.4. we obtain that $\pi(S) \in \mathcal{C}^{*}(V, \delta)$ in $Z$. Since $F:=\left.P\right|_{Z}$ is a linear isomorphism with $F(\pi(S))=P(S)$, Lemma 4.2 implies that $P(S) \in \mathcal{C}^{*}(D, \varepsilon)$ for $D:=F(V)$ and some $\varepsilon>0$. Consequently, $P(S)$ is Aronszajn null in $Y$ by Lemma 4.3. Thus, $\operatorname{int} P(S)=\emptyset$. Since $P(S)$ is an $F_{\sigma}$ set, we obtain that $P(S)$ is a first category set.

As an immediate consequence, we obtain the following result.

Proposition 4.6. Let $X$ be a separable infinite dimensional Banach space, $n \geq 2$, $A \in \mathcal{L}^{n}(X)$, and let $P: X \rightarrow Y$ be a continuous linear mapping onto a Banach space $Y$ such that $\operatorname{dim}(\operatorname{ker}(P))<n$. Then $P(S)$ is a subset of a set from $\mathcal{C}_{n}^{*}$ in $Y$. Consequently, $P(S)$ is a first category subset of $Y$ which is a subset of an Aronszajn null set in $Y$.

Remark 4.7. Let $X, Y, P$ and $n$ be as in Proposition 4.6.

(i) Let $f$ be a continuous convex function on $X$ and $B_{n}:=\{x \in X: \operatorname{dim}(\partial f(x)) \geq$ $n$ \}. Then [13, Theorem 1.3.] states that $P(A)$ is a first category set. Using results of [19, it is easy to see that [13, Theorem 1.3.] is equivalent to the statement that $P(A)$ is a first category for each $A \in \mathcal{D C}^{n}(X)$, but the proof of [13] is direct, it does not use [19].

(ii) The result that $P(A)$ is a first category for each $A \in \mathcal{L}^{n}(X)$ is due to Heisler [7.

(iii) An example from [7] shows that there exists $A \in \mathcal{D C}^{n}(X)$ such that $P(A) \notin$ $\mathcal{L}^{1}(Y)$.

(iv) It is not known whether $P(A)$ is $\sigma$-porous or $\Gamma$-null for each $A \in \mathcal{L}^{n}(X)$ (resp. $\left.A \in \mathcal{D C}^{n}(X)\right)$. The negative answer seems to be probable.

Remark 4.8. Let $X$ be a separable infinite dimensional space. Proposition 4.6 easily implies that the inclusions $\mathcal{L}^{n}(X) \subset \mathcal{L}^{n-1}(X)(n>1)$ are proper. Indeed, no Lipschitz surface $S$ of codimension $n-1$ can belong to $\mathcal{L}^{n}(X)$, since there is a surjective continuous linear projection of $S$ on a space $E$ of codimension $n-1$.

Proposition 4.6 implies the following result which improves both [13, Theorem 1.3.] and [7, Theorem 5.6.]. 
Theorem 4.9. Let $X$ be a separable infinite dimensional space, $n \geq 2$, and let $T: X \rightarrow X^{*}$ be a monotone (mutivalued) operator. Denote by $B_{n}$ the set of all $x \in X$ for which the convex cover of $T(x)$ is at least $n$-dimensional. Let $P: X \rightarrow Y$ be a continuous linear mapping onto a Banach space $Y$ such that $\operatorname{dim}(\operatorname{ker}(P))<n$. Then $P\left(B_{n}\right)$ is a subset of a set from $\mathcal{C}_{n}^{*}$ in $Y$. Consequently, $P\left(B_{n}\right)$ is a first category subset of $Y$ which is a subset of an Aronszajn null set in $Y$.

Proof. Since $B_{n} \in \mathcal{L}^{n}(X)$ by [18, the assertion follows from Proposition 4.6.

Acknowledgements. The research was supported by the institutional grant MSM 0021620839 and by the grant GAČR 201/06/0198.

\section{REFERENCES}

[1] B. Berkson, Some metrics on the subspaces of a Banach space, Pacific J. Math. 13 (1963), 7-22. Zbl 0118.10402

[2] Y. Benyamini, J. Lindenstauss, Geometric Nonlinear Functional Analysis, Vol. 1 Colloqium publications (American Mathematical Society); v. 48, Providence, Rhode Island, 2000. Zbl 0946.46002

[3] J. Duda, On inverses of $\delta$-convex mappings, Comment. Math. Univ. Carolin. 42 (2001), 281-297. Zbl 1053.47522

[4] P. Erdös, On the Hausdorff dimension of some sets in Euclidean space, Bull. Amer. Math. Soc. 52 (1946), 107-109. Zbl 0063.01272

[5] I.C. Gochberg, M.G. Krein, Fundamental aspects of defect numbers, root numbers, and indexes of linear operators, Uspekhi Mat. Nauk 12 (1957), 43-118 (in Russian). Zbl 0088.32101

[6] P. Hartman, On functions representable as a difference of convex functions, Pacific J. Math. 9 (1959), 707-713. Zbl 0093.06401

[7] M. Heisler, Some aspects of differentiability in geometry on Banach spaces, Ph.D. thesis, Charles University, Prague, 1996.

[8] T. Kato, Perturbation Theory for Linear Operators, Springer-Verlag, Berin, 1976. Zbl 0342.47009

[9] E. Kopecká, J. Malý, Remarks on delta-convex functions, Comment. Math. Univ. Carolin. 31 (1990), 501-510. Zbl 0714.46007

[10] A. Largillier, A note on the gap convergence, Appl. Math. Lett. 7 (1994), 67-71. Zbl 0804.46026

[11] J. Lindenstrauss, D. Preiss, Fréchet differentiability of Lipschitz functions (a survey), in: Recent Progress in Functional Analysis, 19-42, North-Holland Math. Stud. 189, North-Holland, Amsterdam, 2001. Zbl 1037.46043

[12] J. Lindenstrauss, D. Preiss, On Fréchet differentiability of Lipschitz maps between Banach spaces, Annals Math. 157 (2003), 257-288. Zbl pre01933158

[13] D. Preiss, Almost differentiability of convex functions in Banach spaces and determination of measures by their values on balls, Collection: Geometry of Banach spaces (Strobl, 1989), 237-244, London Math. Soc. Lecture Note Ser. 158, 1990. Zbl 0758.46034

[14] D. Preiss, L. Zajíček, Directional derivatives of Lipschitz functions, Israel J. Math. 125 (2001), 1-27. Zbl 1022.46026

[15] L. Veselý, On the multiplicity points of monotone operators on separable Banach spaces, Comment. Math. Univ. Carolin. 27 (1986), 551-570. Zbl 0616.47043

[16] L. Veselý, L. Zajíček, Delta-convex mappings between Banach spaces and applications, Dissertationes Math. (Rozprawy Mat.) 289 (1989), 52 pp. Zbl 0685.46027

[17] L. Zajíček, On the points of multivaluedness of metric projections in separable Banach spaces, Comment. Math. Univ. Carolin. 19 (1978), 513-523. Zbl 0382.46007

[18] L. Zajíček, On the points of multiplicity of monotone operators, Comment. Math. Univ. Carolin. 19 (1978), 179-189. Zbl 0404.47025

[19] L. Zajíček, On the differentiation of convex functions in finite and infinite dimensional spaces, Czechoslovak Math. J. 29 (1979), 340-348. Zbl 0429.46007 
[20] L. Zajíček, Differentiability of the distance function and points of multi-valuedness of the metric projection in Banach space, Czechoslovak Math. J. 33(108)(1983), 292-308. Zbl 0527.41028

[21] L. Zajíček, On $\sigma$-porous sets in abstract spaces, Abstract Appl. Analysis 2005 (2005), 509-534. Zbl pre05038297

Authors adress: Luděk Zajiček, Charles University, Faculty of Mathematics and Physics, Sokolovská 83, 18675 Praha 8, Czech Republic, e-mail: zajicek@karlin.mff.cuni.cz. 